\title{
Tension arises from duality at the heart of taxonomy
}

\section{Names must both represent a volatile hypothesis and provide a key to lasting information.}

Sir-H. C. J. Godfray (Nature 417, 17-19; 2002) points out in his Commentary, quite rightly, that taxonomy and systematics, the naming and classification of living things, have an image problem among funding bodies and the community in general. He says the discipline faces a crisis: much information on living things needs to be captured, organized and disseminated, but funds often go into higher-profile projects such as the Sloan Digital Sky Survey and the Human Genome Project.

What do these projects offer that taxonomy does not? One thing is a relatively centralized model of data organization and retrieval. One can enter a web portal and easily find and download an authoritative account of star positions or gene sequences. No equivalent portals currently exist for taxonomic information (although initiatives are in place to provide them: see Nature 418, 362-363 \& 367; 2002). To create such a portal, Godfray suggests that taxonomy needs to move from the current dispersed, decentralized approach to a unitary, centralized one.

Although we agree that Godfray's model would provide many advantages to outside users, we believe that it misses one crucial problem. A taxon - the base unit of a taxonomy - is an hypothesis, not an observation or "fact". It is not like a star or a base pair, and taxonomy is not like a star survey or genome project. Because of this, solutions cannot be so easily transferred from these domains to taxonomy.

Taxonomists routinely filter large arrays of observations through an extensive knowledge base to decide that this group of specimens or that set of prior taxa comprises a new taxon. Crucially, another taxonomist using the same knowledge base may validly arrive at quite different conclusions, and it may take time for thorough testing of the alternative concepts to arrive at consensus (itself subject to future challenge and refinement).

Taxonomy is a complex and dynamic science, not a simple (albeit technically difficult) collation of facts like the nucleotide sequences of a genome survey. It is probably only at the most complex levels of genetics, in dynamic proteomics or developmental biology,

that anything like the complexity of hypothesis-generation and testing involved in routine taxonomy and systematics is encountered.

Any proposal for changing the processes of taxonomy must account for this. Godfray's model, in which new names require validation by a central committee before acceptance, could lead to authoritarianism and a stifling of innovative taxonomic viewpoints. No other hypothesis-driven field of science would accept such a straitjacket.

The heart of the problem is the central duality and tension in taxonomic names. On the one hand, names are convenient shorthand representations of scientific hypotheses, and as such should be as volatile as hypotheses in any other field proposed, used, modified and then perhaps discarded as evidence dictates. On the other hand, taxonomic names are keys to biological information, unique codes that unlock the library of knowledge about those organisms, and for this purpose they must be non-volatile or the links will be lost. A solution must adequately recognize these dual roles and decouple the system that allows maximum freedom of hypothesis-generation from the system that provides names for users. Kevin Thiele, David Yeates

CSIRO Plant Industry and Entomology, Commonwealth Scientific and Industrial Research Organisation, Canberra, Australia

\section{No alternative to animal tests for behaviour}

Sir - Your News story "Race is on to find alternative to animal tests" (Nature 418, $116 ; 2002)$ discussed the possibility that in vitro methods could replace animal tests used in the toxicologic screening of chemicals. However, it is highly unlikely that in vitro approaches could be used to replace in vivo animal tests in the assessment of neurotoxicity. For instance, behavioural assessment in rodents is a powerful experimental tool in the evaluation of the effects of chemicals on neural function. Pharmaceutical companies and regulatory agencies consider behavioural toxicology an important part of the screening of new pharmaceutical products and potentially neurotoxic chemicals.

It is true that in vitro experimental approaches can be used in the investigation of the cellular and molecular mechanisms of behaviour. But druginduced alterations in parameters such as anxiety, motor activity, and learning can only be assessed by in vivo tests on mammals. It is not possible to replace such tests with non-animal methods using, for example, human cells or yeast. Rafael Roesler Genotoxicity Laboratory, Centre for Biotechnology, Federal University of Rio Grande do Sul, 91501-970 Porto Alegre, RS, Brazil

\section{Laws stay constant but the world changes}

Sir - Efforts by Stephen Schneider and Richard Moss to quantify uncertainty (Nature 418, 476-478; 2002) succeeded with the Intergovernmental Panel on Climate Change (IPCC) Working Group 1 climate modellers but failed with Working Group 3's emissions modellers. As a lead author on the IPCC's 2000 Special Report on Emissions Scenarios, I would like to elaborate on why this happened.

The first group of scientists know that the laws of physics operative today will be the same laws of physics operative in 2100 . The second group, of economists and analysts, know with equal certainty that the geopolitical, socioeconomic and technological forces operative today are likely to be radically different in 2100 .

\section{Stuart R. Gaffin}

Center for International Earth Science Information Network (CIESIN), Columbia University, 61 Route 9W, PO Box 1000, Palisades, New York 10964, USA

\section{Food labels should state the benefits of GMOs}

Sir - You state in the News story "Europe gets tough on labelling genetically modified foodstuffs" (Nature 418, 114; 2002) that an angry food industry claims that stricter labels would imply a health risk.

On the contrary. The GMOs potentially present in trace amounts have been far more thoroughly assessed for safety than the rest of the food, but the labelling tells us nothing of this. The European Union has selected GMOs for special labelling because many people are uncertain of the benefits and risks. Surely, then, labels should convey the positive (and, where appropriate, negative) environmental, nutritional or economic attributes of the modification? This would help consumers make informed decisions to buy or avoid products.

Renton Righelato

63 Hamilton Road, Reading RG1 5RA, UK 